\title{
Carbon C 11 Metformin
}

National Cancer Institute

\section{Source}

National Cancer Institute. Carbon C 11 Metformin. NCI Thesaurus. Code C130014.

A radiopharmaceutical tracer containing the anti-diabetic agent metformin, a biguanide and hydrophilic organic cation, and labeled with carbon 11 (11C), used to measure metformin uptake by cancer cells using positron emission tomography (PET). Upon administration of carbon C 11 metformin, the metformin moiety targets and binds to cancer cells; in turn, the compound is taken up by cancer cells. Upon PET, metformin distribution into cancer cells can be visualized, which allows for a prediction of the anticancer efficacy of metformin, based on tumor cell uptake. Metformin transport, such as uptake into cancer cells, appears to be correlated with the expression of org anic cation transporters (OCT ) 1-3, multidrug and toxin extrusion (MATE) 1 and 2, and plasma membrane monoamine transporter (PMAT). OCTs, MATEs and PMAT play a key role in the distribution and excretion of organic cationic compounds. 